\title{
Bending Analysis of Curved Orthotropic Plate and Its Application in Curved Bridges on Slope
}

\author{
Shuchen HU \\ School of Highway, Chang' an University \\ Xi'an 710064, Shaanxi, China \\ CCCC RUITONG Road \& Bridge Maintenance \\ Technology Co., Ltd, Xi'an 710075, Shaanxi, China \\ E-mail: shuchenhu@126.com
}

\author{
Zhenjia WANG \\ School of Highway, Chang'an University \\ Xi'an 710064, Shaanxi, China \\ E-mail: asdwangzhenjia@163.com
}

Dalin HU

School of Highway, Chang'an University

Xi' an 710064, Shaanxi, China

E-mail: dlhu@gl.chd.edu.cn

\begin{abstract}
Based on the Kirchhoff's thin plate hypothesis and static equilibrium of element, the governing differential equations of curved orthotropic plate in polar coordinates which is subjected to normal and tangential surface load are derived. According to the governing differential equations and boundary conditions, analytical solutions of displacements and internal forces for a simple supported curved plate on slope(longitudinal gradient is $\beta$ ) under vertical uniform distribution load are obtained. With the computer program based on the formula, the results of the displacements and internal forces for the bridge under given conditions are achieved. The results showed that the bending behavior was not affected by the tangential components when $\beta$ is small. The differential equations for bending analysis and their solutions represent a new way for describing a simple supported curved bridge on slope.
\end{abstract}

Keywords-curved bridge on slope; curved orthotropic plate; bending analysis; the static equilibrium equation; analytical solution; numerical solution

\section{INTRODUCTION}

There are a lot of studies[1] on the application of the theory of curved orthotropic plate for the analysis of curved bridge, but the different studies are somewhat similar for the complication of the problem and the detailed report[2] still took the hypotheses of $\mu=\sqrt{\mu_{\gamma} \mu_{\theta}}$ and $D_{\gamma \theta}=\sqrt{D_{\gamma} D_{\theta}}$. Chinese scholars have made thorough studies[3,4,5] on the simply supported curved bridge of ribbed plate with the theory of comparative orthotropic curved plate in which the hypotheses $\mu_{\gamma}=\mu_{\theta}=0$ were taken. In this paper, based on the static equilibrium condition, the bending differential equations of the curved orthotropic plate under normal and tangential loads are obtained and the bending problem for the curved bridge on slope under vertical loads is solved with the equations.

\section{GOVERNING EQUATIONS AND BOUNDARY CONDITIONS}

Drawing a parallel between the superstructure of curved bridges on slope and the orthotropic plate according with Kirchhoff's. hypotheses to solve the problem of curved plate quasi-static equilibrium. To solve the problem of the curved bridge on slope, first we select the Cartesian coordinates xyz and let the middle surface coincide with the xoy plane and replace the tangential surface forces of the plate with the forces on the middle surface and the moments, and propose a free body diagram as shown in Fig. 1. The governing equations are established with the static equilibrium of the forces and the moments in different direction, after rearrangement we have,

$$
\sum F_{\mathrm{x}^{\prime}}=0 \frac{\partial N_{r}}{\partial r}+\frac{1}{r} \frac{\partial N_{r \theta^{\prime}}}{\partial \theta}+\frac{N_{r}-N_{\theta}}{r}+q_{t r}=0
$$

$$
\begin{gathered}
\sum F_{\mathrm{y}}=0 \quad \frac{1}{r} \frac{\partial N_{\theta}}{\partial \theta}+\frac{\partial N_{\theta r}}{\partial r}+\frac{2 N_{r \theta}}{r}+q_{r \theta}=0 \\
\sum M_{z}=0 \quad N_{\theta r}=N_{r \theta} \\
\sum F_{z}=0 \quad \frac{1}{r} \frac{\partial Q_{\theta}}{\partial \theta}+\frac{\partial Q_{\theta r}}{\partial r}+\frac{Q_{r}}{r}+q_{n}=0 \\
\sum M_{x}=0 \quad \frac{\partial M_{r \theta}}{\partial r}+\frac{1}{r} \frac{\partial M_{\theta}}{\partial \theta}+\frac{M_{r \theta}+M_{\theta r}}{r}-\frac{h}{2} q_{r \theta}-Q_{\theta}=0
\end{gathered}
$$


$\sum M_{y}=0 \frac{\partial M_{r}}{\partial r}+\frac{1}{r} \frac{\partial M_{\theta r}}{\partial \theta}+\frac{M_{r}-M_{\theta}}{r}-\frac{h}{2} q_{t r}-Q_{r}=0$



$I^{2}$
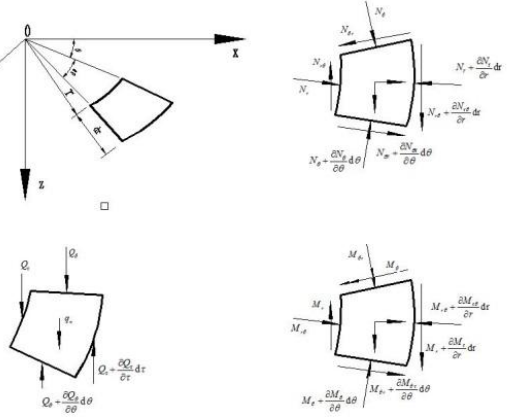

Figure 1. Plate Elements and Element Forces

Substituting equation [3] into equation [2], obtain

$$
\frac{\partial N_{r \theta}}{\partial r}+\frac{1}{r} \frac{\partial N_{\theta}}{\partial \theta}+\frac{2 N_{r \theta}}{r}+q_{\tau \theta}=0
$$

Equation [1] and equation [2'] are the static equilibrium equations of the curved bridge under the forces on the middle and the solution for the plane stress problem can be given from the equations with coordinate compatibility conditions and boundary conditions[6], which are not discussed here.

From equations [5] and [6], obtain $\frac{\partial Q_{r}{ }^{3}}{\partial r}, \frac{Q_{r}}{r}$ and $\frac{1}{r} \frac{\partial Q_{\theta}}{\partial \theta}$, substituted into equation [4], give

$$
\begin{aligned}
& \frac{\partial^{2} M_{r}}{\partial r^{2}}+\frac{1}{r^{2}} \frac{\partial^{2} M_{\theta}}{\partial \theta^{2}}+\frac{1}{r}\left(\frac{\partial^{2} M_{r \theta}}{\partial r \partial \theta}+\frac{\partial^{2} M_{\theta r}}{\partial r \partial \theta}\right)+\frac{1}{r^{2}}\left(\frac{\partial M_{r \theta}}{\partial \theta}+\frac{\partial M_{\theta \gamma}}{\partial \theta}\right)+\frac{2}{r} \frac{\partial M_{\gamma}}{\partial r} \\
& -\frac{1}{r} \frac{\partial M_{\theta}}{\partial r}-\frac{h}{2}\left(\frac{\partial q_{\tilde{r}}}{\partial r}+\frac{1}{r} q_{\nabla}+\frac{1}{r} \frac{\partial q_{r}}{\partial \theta}\right)+q_{n}=0
\end{aligned}
$$

In the polar coordinates the expression[7] for the internal forces and reactions of the orthotropic plate are

$$
\begin{gathered}
M_{r}=-D_{r}\left[\frac{\partial^{2} W}{\partial r^{2}}+\mu_{\theta}\left(\frac{1}{r} \frac{\partial W}{\partial r}+\frac{1}{r^{2}} \frac{\partial^{2} W}{\partial r^{2}}\right)\right] \\
M_{\theta}=-D_{\theta}\left[\frac{1}{r} \frac{\partial W}{\partial r}+\frac{1}{r^{2}} \frac{\partial^{2} W}{\partial \theta^{2}}+\mu_{r} \frac{\partial^{2} W}{\partial r^{2}}\right] \\
M_{r \theta}=-2 D_{k}\left[\frac{1}{r} \frac{\partial^{2} W}{\partial r \partial \theta}-\frac{1}{r^{2}} \frac{\partial W}{\partial \theta}\right] \\
M_{r \theta}=M_{\theta r}
\end{gathered}
$$

$$
\begin{gathered}
Q_{r}=-\left[D_{r}\left(\frac{\partial^{3} W}{\partial r^{3}}+\frac{1}{r} \frac{\partial^{2} W}{\partial r^{2}}\right)+D_{r \theta}\left(\frac{1}{r^{2}} \frac{\partial^{2} W}{\partial r \partial \theta}+\frac{1}{r^{3}} \frac{\partial^{3} W}{\partial \theta^{2}}\right)-D_{\theta}\left(\frac{1}{r^{2}} \frac{\partial W}{\partial r}+\frac{1}{r^{3}} \frac{\partial^{2} W}{\partial \theta^{2}}\right)\right] \\
Q_{\theta}=-\left[D_{r \theta} \frac{1}{r} \frac{\partial^{3} W}{\partial r^{2} \partial \theta}+D_{\theta}\left(\frac{1}{r^{2}} \frac{\partial^{2} W}{\partial r \partial \theta}+\frac{1}{r^{3}} \frac{\partial^{3} W}{\partial \theta^{3}}\right)\right] \\
V_{r}=Q_{r}+\frac{1}{r} \frac{\partial M_{r \theta}}{\partial \theta}=Q_{r}-2 D_{k}\left(\frac{1}{r^{2}} \frac{\partial^{3} W}{\partial r \partial \theta^{2}}-\frac{1}{r^{3}} \frac{\partial^{3} W}{\partial \theta^{3}}\right) \\
V_{\theta}=Q_{\theta}+\frac{\partial M_{\theta r}}{\partial r}=Q_{\theta}-2 D_{k}\left(\frac{1}{r} \frac{\partial^{3} W}{\partial r^{2} \partial \theta}-\frac{2}{r^{2}} \frac{\partial^{2} W}{\partial r \partial \theta}+\frac{2}{r^{3}} \frac{\partial W}{\partial \theta}\right)
\end{gathered}
$$

Where

$$
D_{r}=\frac{E_{r} h^{3}}{12\left(1-\mu_{r} \mu_{\theta}\right)} \quad D_{\theta}=\frac{E_{\theta} h^{3}}{12\left(1-\mu_{r} \mu_{\theta}\right)} \quad D_{k}=\frac{G h^{3}}{12}
$$

Where $\mathrm{E}_{\mathrm{r}}, \mathrm{E}_{\theta}, \mu_{\mathrm{r}}$ and $\mu_{\theta}$ are the elastic modular of material in direction $\mathrm{r}, \theta$ and Poisson's ratio respectively and $\mathrm{G}$ is modulus of shearing and $\mathrm{h}$ is thickness of plate. Substituting [7] [10] into equation [4'], obtain

$$
\begin{aligned}
& D_{r} \frac{\partial^{4} W}{\partial r^{4}}+\frac{D_{r} \mu_{\theta}+D_{\theta} \mu_{r}+4 D_{k}}{r^{2}} \frac{\partial^{4} W}{\partial r^{2} \partial \theta^{2}}+D_{\theta} \frac{1}{r^{4}} \frac{\partial^{4} W}{\partial \theta^{4}} \\
& +\frac{2 D_{r}+D_{r} \mu_{\theta}-D_{\theta} D_{r}}{r} \frac{\partial^{3} W}{\partial r^{3}}-\frac{2 D_{r} \mu_{\theta}+4 D_{k}}{r^{3}} \frac{\partial^{3} W}{\partial r \partial \theta^{2}} \\
& +\frac{2 D_{\theta}+2 D_{r} \mu_{\theta}+4 D_{k}}{r^{4}} \frac{\partial^{2} W}{\partial \theta^{2}}+D_{\theta} \frac{1}{r^{3}} \frac{\partial W}{\partial r}-D_{\theta} \frac{1}{r^{2}} \frac{\partial^{2} W}{\partial^{2}} \\
& =q_{n}-\frac{h}{2}\left(\frac{\partial q_{\pi}}{\partial r}+\frac{1}{r} q_{r}+\frac{1}{r} \frac{\partial q_{\theta}}{\partial \theta}\right)
\end{aligned}
$$

Let $2 D_{r}=D_{r} \mu_{\theta}+D_{\theta} \mu_{r}+4 D_{k}$, and note that the normal practice in the bending analysis of orthotropic plate takes $D_{r} \mu_{\theta}=D_{\theta} \mu_{r}$, hence equation [4', $]$ is simplified as.

$$
\begin{aligned}
& D_{r} \frac{\partial^{4} W}{\partial r^{4}}+2 D_{r \theta} \frac{1}{r^{2}} \frac{\partial^{4} W}{\partial r^{2} \partial \theta^{2}}+D_{\theta} \frac{1}{r^{4}} \frac{\partial^{4} W}{\partial \theta^{4}}+2 D_{r} \frac{1}{r} \frac{\partial^{3} W}{\partial r^{3}} \\
& -2 D_{r \theta} \frac{1}{r^{3}} \frac{\partial^{3} W}{\partial r \partial \theta^{2}}+2\left(D_{\theta}+D_{r \theta}\right)+\frac{1}{r^{4}} \frac{\partial^{2} W}{\partial \theta^{2}}+D_{\theta} \frac{1}{r^{3}} \frac{\partial W}{\partial r} \\
& -D_{\theta} \frac{1}{r^{2}} \frac{\partial^{2} W}{\partial r^{2}}=q_{n}-\frac{h}{2}\left(\frac{\partial q_{\vartheta}}{\partial r}+\frac{1}{r} q_{\theta}+\frac{1}{r} \frac{\partial q_{t \theta}}{\partial \theta}\right)
\end{aligned}
$$

Considering simply supported curved bridge on slope of single span, the boundary conditions for equation [15] are that two radial sides are simply supported and two curved sides are supported free, i.e.

For the simply supported sides, we have 


$$
\left.\begin{array}{l}
\left.W(r, \theta)\right|_{\theta=0}=0 \\
\left.M_{\theta}(r, \theta)\right|_{\theta=0}=0 \\
\left.M_{\theta}(r, \theta)\right|_{\theta=\varphi}=0
\end{array}\right\}
$$

For the free side, we have

$$
\left.\begin{array}{cc}
\left.M(r, \theta)\right|_{r=a}=0 & \left.M_{r}(r, \theta)\right|_{r=b}=0 \\
\left.V_{r}(r, \theta)\right|_{r=a}=0 & \left.V_{r}(r, \theta)\right|_{r=b}=0
\end{array}\right\}
$$

\section{SOlution OF THE PROBlem}

\section{A. Solution for Homogeneous Equations}

The homogeneous equation for equation [15] is

$$
\begin{aligned}
& D_{r} \frac{\partial^{4} W}{\partial r^{4}}+2 D_{r \vartheta} \frac{1}{r^{2}} \frac{\partial^{4} W}{\partial^{2} \partial \theta^{2}}+D_{\theta} \frac{1}{r^{4}} \frac{\partial^{4} W}{\partial \theta^{4}}+2 D_{r} \frac{1}{r} \frac{\partial^{3} W}{\partial r^{3}}-2 D_{r \theta} \frac{1}{r^{3}} \frac{\partial^{3} W}{\partial r \partial \theta^{2}} \\
& +2\left(D_{\theta}+D_{r \theta}\right)+\frac{1}{r^{4}} \frac{\partial^{2} W}{\partial \theta^{2}}+D_{\theta} \frac{1}{r^{3}} \frac{\partial W}{\partial r}-D_{\theta} \frac{1}{r^{2}} \frac{\partial^{2} W}{\partial r^{2}}=0
\end{aligned}
$$

The general solution for the homogeneous equation is

$$
W_{0}(r, \theta)=\sum_{n=1}^{\infty} R_{n}(r) \sin \frac{n \pi \theta}{\varphi}
$$

Substituting the expression above into equation [18], give

$$
R_{n}^{(4)}(r)+\frac{2}{r} R_{n}^{\prime \prime}(r)-Z \frac{1}{r^{2}} R_{n}^{\prime \prime}(r)+Z \frac{1}{r^{3}} R_{n}^{\prime}(r)+Y \frac{1}{r^{4}} R_{n}(r)=0
$$

Where

$$
\begin{aligned}
& Z=\delta^{2}+2 a \delta \lambda, \quad Y=\delta^{2} \lambda^{2}-\left(2 \delta^{2}+2 a \delta\right) \lambda^{2} \\
& \delta=\sqrt{D_{\theta} / D_{r}}, \quad a \delta=D_{r \theta} / D_{r}, \quad \lambda=n \pi / \varphi
\end{aligned}
$$

Let $R_{n}(r)=r^{T}$ and substitute into equation [19], obtain the roots of characteristic equation

$$
T_{1,2,3,4}=1 \pm \sqrt{\left(\frac{1+\delta}{2}\right)^{2}+\frac{\alpha-1}{2} \delta \lambda^{2}} \pm \sqrt{\left(\frac{1-\delta}{2}\right)^{2}+\frac{\alpha+1}{2} \delta \lambda^{2}}
$$

Hence

$$
W_{0}(r, \theta)=\sum_{n=1}^{\infty}\left(\sum_{i=1}^{4} C_{n}^{1 i} r^{T i}\right) \sin \frac{n \pi \theta}{\varphi}
$$

B. Particular solution under vertical uniform distribution load

For the coordinates system shown in Fig. 2, the normal and tangential loads of plate under u.d.l. $\mathrm{q}$ are

$$
\left.\begin{array}{c}
q_{n}=q \cos \beta \\
q_{\tau r}=-q \sin \beta \sin \theta
\end{array}\right\}
$$

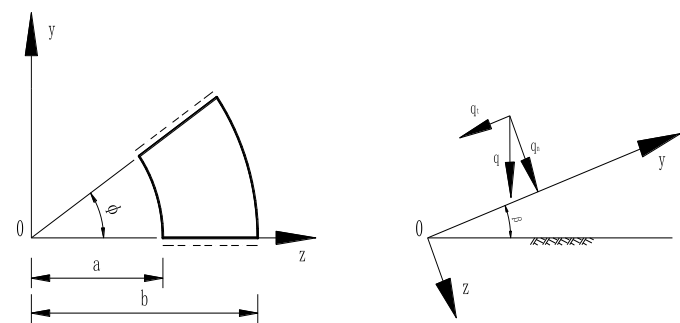

Figure 2. General coordinates for plate bridge

Substituting expression [22] into equation [15], gives

$$
\begin{aligned}
& D_{r} \frac{\partial^{4} W}{\partial r^{4}}+2 D_{r \theta} \frac{1}{r^{2}} \frac{\partial^{4} W}{\partial r^{2} \partial \theta^{2}}+D_{\theta} \frac{1}{r^{4}} \frac{\partial^{4} W}{\partial \theta^{4}}+2 D_{r} \frac{1}{r} \frac{\partial^{3} W}{\partial r^{3}} \\
& -2 D_{r \theta} \frac{1}{r^{3}} \frac{\partial^{3} W}{\partial r \partial \theta^{2}}+2\left(D_{\theta}+D_{r \theta}\right)+\frac{1}{r^{4}} \frac{\partial^{2} W}{\partial \theta^{2}}+D_{\theta} \frac{1}{r^{3}} \frac{\partial W}{\partial r} \\
& -D_{\theta} \frac{1}{r^{2}} \frac{\partial^{2} W}{\partial r^{2}}=q \cos \beta
\end{aligned}
$$

Assume the solution satisfied the boundary condition for radial sides is

$$
W_{1}(r, \theta)=\sum_{n=1}^{\infty} D_{n}^{1} r^{4} \sin \frac{n \pi \theta}{\varphi}
$$

Substituting expression [24] into equation [23] and using orthogonality of sine series, obtain

$$
\left.\begin{array}{c}
D_{n}^{1}=\frac{2 q \cos \beta(1-\cos n \pi)}{n \pi \varphi(72-8 Z+Y) D_{r}} \\
W_{r}(r, \theta)=\sum_{n=1}^{\infty} \frac{2 q \cos \beta(1-\cos n \pi)}{n \pi \varphi(72-8 Z+Y) D_{r}} r^{4} \sin \frac{n \pi \theta}{\varphi}
\end{array}\right\}
$$

\section{General Solution of the Equation and the Expression for Internal Forces}

Under the vertical u.d.l. q, the general solution for equation [15] is $W(r, \theta)=W_{0}(r, \theta)+W_{1}(r, \theta)$. i.e.

$$
W(r, \theta)=\sum_{n=1}^{\infty}\left[\sum_{i=1}^{4} C_{n}^{1 i} r^{T i}+D_{n}^{1} r^{4}\right] \sin \frac{n \pi \theta}{\varphi}
$$

Let $r=\rho r_{0} \quad C_{n}^{i}=C_{n}^{1 i} r_{0}^{T_{i}} \quad D_{n}=D_{n}^{1} r_{0}^{4}$, where $r_{0}=a+(b-a) / 2$, then equation [25] can be expressed as

$$
W(r, \theta)=\sum_{n=1}^{\infty}\left[\sum_{i=1}^{4} C_{n}^{i} \rho^{T_{i}}+D_{n}^{1} \rho^{4}\right] \sin \frac{n \pi \theta}{\varphi}
$$

From equation [25], we can find displacement partial derivative for computing internal forces and reactions.

$$
\text { Let } \frac{\partial^{i+j} W}{\partial r^{i} \partial \theta^{j}}=W(r, \theta) \text {, obtain }
$$




$$
\begin{aligned}
& W(1,0)=\frac{1}{r_{0}} \sum_{n=1}^{\infty}\left[\sum_{i=1}^{4} C_{n}^{i} T_{i} \rho^{T_{i}-1}+4 D_{n} \rho^{4}\right] \sin \frac{n \pi \theta}{\varphi} \\
& W(2,0)=\frac{1}{r_{0}^{2}} \sum_{n=1}^{\infty}\left[C_{n}^{i} T_{i}\left(T_{i}-1\right) \rho^{T_{i}-2}+2 D_{n} \rho^{2}\right] \sin \frac{n \pi \theta}{\varphi} \\
& W(3,0)=\frac{1}{r_{0}^{3}} \sum_{n=1}^{\infty}\left[\sum_{i=1}^{4} C_{n}^{i} T_{i}\left(T_{i}-1\right)\left(T_{i}-2\right) \rho^{T_{i}-3}+24 D_{n} \rho\right] \sin \frac{n \pi \theta}{\varphi} \\
& W(0,1)=\sum_{n=1}^{\infty}\left[\sum_{i=1}^{4} C_{n}^{i} \rho^{T_{i}}+D_{n} \rho^{4}\right] \frac{n \pi}{\varphi} \cos \frac{n \pi \theta}{\varphi} \\
& W(0,2)=\sum_{n=1}^{\infty}\left[\sum_{i=1}^{4} C_{n}^{i} \rho^{T_{i}}+D_{n} \rho^{4}\right]\left(-\frac{n^{2} \pi^{2}}{\varphi^{2}}\right) \sin \frac{n \pi \theta}{\varphi} \\
& W(0,3)=\sum_{n=1}^{\infty}\left[\sum_{i=1}^{4} C_{n}^{i} \rho^{T_{i}}+D_{n} \rho^{4}\right]\left(-\frac{n^{3} \pi^{3}}{\varphi^{3}}\right) \cos \frac{n \pi \theta}{\varphi} \\
& W(1,1)=\frac{1}{r_{0}} \sum_{n=1}^{\infty}\left[\sum_{i=1}^{4} C_{n}^{i} T_{i} \rho^{T_{i}-1}+4 D_{n} \rho^{3}\right] \frac{n \pi}{\varphi} \cos \frac{n \pi \theta}{\varphi} \\
& W(2,1)=\frac{1}{r_{0}} \sum_{n=1}^{\infty}\left[\sum_{i=1}^{4} C_{n}^{i} T_{i}(T-1) \rho^{T_{i}-1}+12 D_{n} \rho^{3}\right] \frac{n^{2} \pi^{2}}{\varphi^{2}} \cos \frac{n \pi \theta}{\varphi} \\
& W(1,2)=\frac{1}{r_{0}} \sum_{n=1}^{\infty}\left[\sum_{i=1}^{4} C_{n}^{i} T_{i} \rho^{T_{i}-1}+4 D_{n} \rho^{3}\right]\left(-\frac{n^{2} \pi^{2}}{\varphi^{2}}\right) \sin \frac{n \pi \theta}{\varphi}
\end{aligned}
$$

Substituting the above expressions into equations [4] [7], give

$$
\begin{aligned}
& M_{r}=-D_{r}\left\{W(2,0)+\mu_{0}\left[W(1,0) / \rho r_{0}+W(0,2) / \rho^{2} r^{2}\right]\right\} \\
& M_{\theta}=-D_{\theta}\left\{W(1,0) / \rho r_{0}+W(0,2) / \rho^{2} r_{0}^{2}+\mu_{r} W(2,0)\right\} \\
& M_{r \theta}=-2 D_{k}\left[W(1,1) / \rho r_{0}-W(0,1) / \rho^{2} r_{0}^{2}\right] \\
& Q_{r}=-\left\{D_{r}\left[W(3,0)+W(2,0) / \rho r_{0}\right]\right. \\
& -D_{\theta}\left[W(1,0) / \rho^{2} r_{0}^{2}+W(0,2) / \rho^{3} r^{3}\right] \\
& \left.+D_{r \theta}\left[W(1,2) \rho^{2} V_{0}^{2}-W(0,2) / \rho^{3} r_{0}^{3}\right]\right\} \\
& Q_{\theta}=-\left\{D_{\theta}\left[W(1,1) / \rho^{2} r_{0}^{2}+W(0,3) / \rho^{3} r_{0}^{3}\right]+D_{r \theta} W(2,1) / \rho r_{0}\right\} \\
& V_{r}=Q_{r}-2 D_{k}\left[W(1,2) / \rho^{2} r_{0}^{2}-W(0,2) / \rho^{3} r_{0}^{3}\right] \\
& V_{\theta}=Q_{r}-2 D_{k}\left[W(2,1) / \rho r_{0}-2 W(1,1) / \rho^{2} r_{0}^{2}+2 W(0,1) / \rho^{3} r_{0}^{3}\right]
\end{aligned}
$$

\section{Determination of Arbitrary Constants $C_{n}^{i}$}

Arbitrary constants $C_{n}^{i}(i=1,2,3,4)$ can be determined by the boundary conditions of the two free sides, if we use dimensionless argument, equation [17] can be expressed as

$$
\left.\begin{array}{lc}
\left.M_{r}\right|_{\rho=a / r_{0}}=0 & \left.M_{r}\right|_{\rho=b / r_{0}}=0 \\
\left.V_{r}\right|_{\rho=a / r_{0}}=0 & \left.V_{r}\right|_{\rho=b / r_{0}}=0
\end{array}\right\}
$$

Let $C_{n}^{i}=X_{i}$, from equation [17'] we have

$$
\left.\begin{array}{c}
X_{1} L_{1}+X_{2} L_{2}+X_{3} L_{3}+X_{4} L_{4}=F_{1} \\
X_{1} L_{5}+X_{2} L_{6}+X_{3} L_{7}+X_{4} L_{8}=F_{2} \\
X_{1} N_{1}+X_{2} N_{2}+X_{3} N_{3}+X_{4} N_{4}=F_{3} \\
X_{1} N_{5}+X_{2} N_{6}+X_{3} N_{7}+X_{4} N_{7}=F_{4}
\end{array}\right\}
$$

Where the coefficients and constant terms

$$
\begin{aligned}
& L_{i}=\left(a / r_{0}\right)^{T_{i}-2}\left(T_{i}^{2}-T_{i}+\mu_{\theta} T_{i}-\mu_{\theta} \frac{n^{2} \pi^{2}}{\varphi^{2}}\right) \\
& L_{i+4}=\left(b / r_{0}\right)^{T_{i-2}}\left(T_{i}^{2}-T_{i}+\mu_{\theta} T_{i}-\mu_{\theta} \frac{n^{2} \pi^{2}}{\varphi^{2}}\right) \\
& \left.N_{i}=\left(a / r_{0}\right)^{T_{i}-3}\left[-D_{r} T_{i}\left(T_{i}-1\right)^{2}+\left(D^{\prime} T_{i}-D^{\prime \prime}\right) \frac{n^{2} \pi^{2}}{\varphi^{2}}+D_{\theta} T_{i}\right]\right\}(i=1,2,3,4) \\
& \left.N_{i+4}=\left(b / r_{0}\right)^{T_{i}-3}\left[-D_{r} T_{i}\left(T_{i}-1\right)^{2}+\left(D^{\prime} T_{i}-D^{\prime \prime}\right) \frac{n^{2} \pi^{2}}{\varphi^{2}}+D_{\theta} T_{i}\right]\right) \\
& F_{1}=-D_{n}\left(a / r_{0}\right)^{2}\left(12+4 \mu_{\theta}-\mu_{\theta} \frac{n^{2} \pi^{2}}{\varphi^{2}}\right) \\
& F_{2}=\left(b / r_{0}\right)^{2} F_{1} \\
& \left.\begin{array}{c}
F_{3}=-D_{n}\left(a / r_{0}\right)^{2}\left[4 D_{\theta}-3 b D_{r}+\left(4 D^{\prime}-D^{\prime \prime}\right) \frac{n^{2} \pi^{2}}{\varphi^{2}}\right] \\
F_{4}=\left(b / r_{0}\right)^{2} F_{3}
\end{array}\right\}
\end{aligned}
$$

Where $D^{\prime}=D_{r 0}+2 D_{k}, \quad D^{\prime \prime}=D^{\prime}+D_{\theta}$

To determine constant terms $X_{i}$, we need to analyze characteristic roots $T_{i}$ in equation [20], let

$$
\begin{array}{ll}
A=\sqrt{\left(\frac{1+\delta}{2}\right)^{2}+\frac{\alpha+1}{2} \delta \lambda^{2}} \quad B=\sqrt{\frac{1-\alpha}{2} \delta \lambda^{2}-\left(\frac{1+\delta}{2}\right)^{2}} \\
S=\sqrt{\left(\frac{1+\delta}{2}\right)^{2}+\frac{\alpha-1}{2} \delta \lambda^{2}} \quad Z=\sqrt{\left(\frac{1+\delta}{2}\right)^{2}+\frac{\alpha-1}{2} \delta \lambda^{2}}
\end{array}
$$

When $Z>A, T_{i}$ are real roots, i.e.

$$
\begin{array}{ll}
T_{1}=1+A+S & T_{2}=1+A-S \\
T_{3}=1-A+S & T_{4}=1-A-S
\end{array}
$$

Substitute $T_{i}$ into equation [27] and equation [28] and then into equations [26] to solve for $X_{i}$, and then substitute $C_{n}^{i}=X_{i}$ into the expression of displacements and internal forces and obtain the solution of the problem.

When $Z<0, T_{i}$ are complex roots, i.e.

$$
\begin{array}{ll}
T_{1}=1+A+i B, & T_{2}=1+A-i B \\
T_{3}=1-A+i B, & T_{4}=1-A-i B
\end{array}
$$

Expressing the coefficients in equation [26] as $x+i y$ and comparing the both sides of the simultaneous equations, we can divide the equations [26] into two simultaneous 
equations according to the principle of equivalence in real parts and imaginary parts, i.e.

$$
\begin{aligned}
& {\left[A^{\prime}\right]\{Z\}=\{F\}} \\
& {\left[A^{\prime \prime}\right]\{Z\}=\{0\}}
\end{aligned}
$$

Where $\left[A^{\prime}\right]$ and $\left[A^{\prime \prime}\right]$ are the matrices of real parts and imaginary parts of $L_{i}$ and $N_{i}$ respectively, $\{F\}$ is the one column matrix of the primary equations. Equations [26"] are homogeneous equations and from which zero and uncertain solution can be obtained. We can determine the constants with equation [26'] and then substitute them into expressions of displacements and internal forces for the solution of the problem. The elements of matrix $\left[A^{\prime}\right]$ are expressed with $L^{\prime}$ and $N^{\prime}$ (Corresponding to $L_{i}$ and $N_{i}$ ) as

$$
\begin{gathered}
L_{1}^{\prime}=\left(a / r_{0}\right)^{A-1}\left\{\cos \left[B \ln \left(a / r_{0}\right)\right] Z A-\sin \left[B \ln \left(a / r_{0}\right)\right] Y A\right\} \\
L_{3}^{\prime}=\left(a / r_{0}\right)^{-A-1}\left\{\cos \left[B \ln \left(a / r_{0}\right)\right] Z B-\sin \left[B \ln \left(a / r_{0}\right)\right] Y B\right\} \\
L_{4}^{\prime}=L_{3}^{\prime}
\end{gathered}
$$

Expressions of $L^{\prime} 5 \sim L^{\prime} 8$ can be obtained with substituting a into $\mathrm{b}$ in expression $L_{1}^{\prime} \sim L_{4}^{\prime}$

$$
\begin{aligned}
& N_{1}^{\prime}=\left(a / r_{0}\right)^{A-2}\left\{\cos \left[B \ln \left(a / r_{0}\right)\right] Z C-\sin \left[B \ln \left(a / r_{0}\right)\right] Y C\right\} \\
& N_{2}{ }^{\prime}=\left(a / r_{0}\right)^{A-2}\left\{\cos \left[B \ln \left(a / r_{0}\right)\right] Z D+\sin \left[B \ln \left(a / r_{0}\right)\right] Y D\right\} \\
& N_{3}{ }^{\prime}=\left(a / r_{0}\right)^{-A-2}\left\{\cos \left[B \ln \left(a / r_{0}\right)\right] Z E+\sin \left[B \ln \left(a / r_{0}\right)\right] Y E\right\} \\
& N_{4}{ }^{\prime}=\left(a / r_{0}\right)^{-A-2}\left\{\cos \left[B \ln \left(a / r_{0}\right)\right] Z F+\sin \left[B \ln \left(a / r_{0}\right)\right] Y F\right\}
\end{aligned}
$$

Expressions of $N^{\prime} 5 \sim N^{\prime} 8$ can be obtained with substituting a into b in expression $N_{1}^{\prime} \sim N^{\prime}{ }_{4}$, where

$$
\begin{aligned}
& Z A=(1+A)^{2}-B^{2}-(1+A)+\mu_{\theta}\left(1+A-\frac{n^{2} \pi^{2}}{\varphi^{2}}\right) \\
& Z B=(1-A)^{2}-B^{2}-(1+A)+\mu_{\theta}\left(1-A-\frac{n^{2} \pi^{2}}{\varphi^{2}}\right) \\
& Z C=-D_{r}\left[(1+A)^{3}-3(1+A) B^{2}+2 B^{2}-2(1+A)^{2}+(1+A)\right] \\
& +\frac{n^{2} \pi^{2}}{\varphi^{2}}\left[D^{\prime}(1+A)-D^{\prime \prime}\right]+D_{\theta}(1+A) \\
& Z D=-D_{r}\left[(1+A)^{3}+3(1+A) B^{2}-2 B^{2}-2(1+A)^{2}+(1+A)\right] \\
& +\frac{n^{2} \pi^{2}}{\varphi^{2}}\left[D^{\prime}(1+A)-D^{\prime \prime}\right]+D_{\theta}(1+A) \\
& Z E=-D_{r}\left[(1-A)^{3}+3(1-A) B^{2}-2(1-A)^{2}+(1-A)-2 B^{2}\right] \\
& +\frac{n^{2} \pi^{2}}{\varphi^{2}}\left[D^{\prime}(1-A)-D^{\prime \prime}\right]+D_{\theta}(1+A) \\
& Z F=-D_{r}\left[(1-A)^{3}+3(1-A) B^{2}-2(1-A)^{2}+(1-A)-2 B^{2}\right]
\end{aligned}
$$

$$
+\frac{n^{2} \pi^{2}}{\varphi^{2}}\left[D^{\prime}(1-A)-D^{\prime \prime}\right]+D_{\theta}(1+A)
$$

$$
Y A=2(1+A) B-B+\mu_{\theta} B \quad Y B=B-2(1-A) B-\mu_{\theta} B
$$

$$
\begin{aligned}
& Y C=-D_{r}\left[(1+A)^{2}+(1+2 B)-(1+B+2 A) B\right] \\
& +B\left(D^{\prime} \frac{n^{2} \pi^{2}}{\varphi^{2}}+D_{\theta}\right) \\
& Y D=-D_{r}\left[(1+A)^{2}+(1-2 B)+(1+B+2 A) B\right] \\
& -B\left(D^{\prime} \frac{n^{2} \pi^{2}}{\varphi^{2}}+D_{\theta}\right) \\
& Y E=-D_{r}\left[(1-A)^{2}+(1-2 B)+(1+B-2 A) B\right] \\
& -B\left(D^{\prime} \frac{n^{2} \pi^{2}}{\varphi^{2}}+D_{\theta}\right) \\
& Y F=-D_{r}\left[(1-A)^{2}+(1+2 B)+(1+B-2 A) B\right] \\
& +B\left(D^{\prime} \frac{n^{2} \pi^{2}}{\varphi^{2}}+D_{\theta}\right)
\end{aligned}
$$

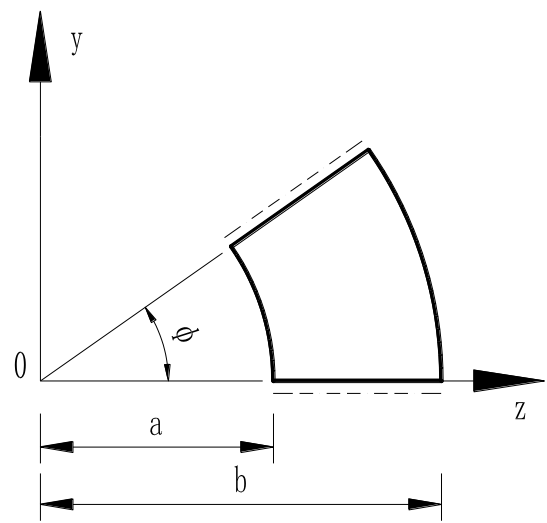

Figure 3. Dimensions of curved bridge

\section{NUMERICAL EXAMPLE}

Fig. 3 shows a simply supported curved plate bridge $(\beta=0)$ subjected to a u.d.l. $\mathrm{q}=1.5 \mathrm{KN} / \mathrm{m}^{2}, \mathrm{Er}=3.0 \times 10^{4} \mathrm{MPa}$, $\mathrm{E}_{\theta}=2.7 \times 10^{4} \mathrm{MPa}, \mu_{1}=0.15, \mathrm{G}=1.22 \times 10^{4} \mathrm{MPa}$, rotational angle $\phi=0.6$. Inner radius $a=21.0 \mathrm{~m}$, outer radius $\mathrm{b}=36.0 \mathrm{~m}$, plate thickness $\mathrm{h}=0.6 \mathrm{~m}$. With the computer program based on the above formula, the displacements and internal forces for the bridge were computed. The displacements and internal forces were plotted as shown in Fig. 4. 

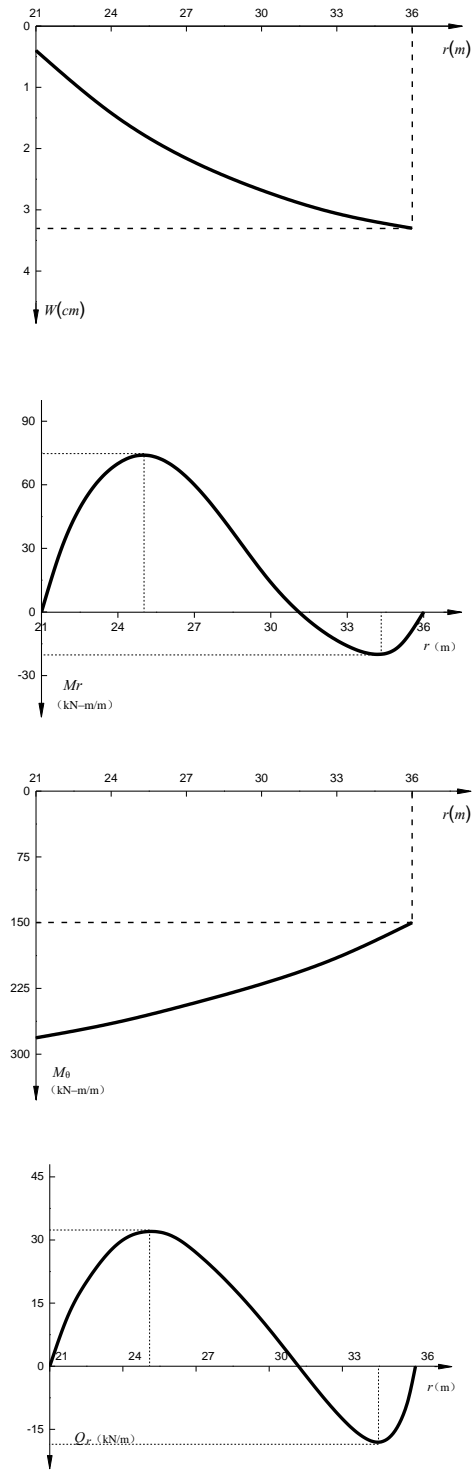

Figure 4. Displacements and internal forces

\section{CONCLUSION}

(1) The deflection differential equations in polar coordinates for orthotropic curved plate under normal and tangential surface loads were derived, which can be used for the bending analysis of material or structure orthotropic curved plate and develop a new way for the computation of curved bridge on slope.

(2) From the solution, the tangential components of loads for curved bridge on slope under vertical u.d.l. do not effect bending behavior of the bridge, since the solution is given under small deformation hypothesis. For the bridge on deeper slope, middle surface force is larger and the interaction between the middle surface force and bending effect must be considered.

(3) The solution can be degenerated into the solution for isotropic curved plate and the approximate solution for orthotropic curved plate in engineering.

(4) In the paper, only simply supported curved plate on slope under vertical u.d.l. is considered. For other boundary conditions (fixed and or continuous to multispan) and complex loading condition, the analysis is different.

\section{ACKNOWLEDGMENT}

This paper is supported by the fund from basic research project of ministry of communications of China (Project No. $2013319812100)$.

\section{REFERENCE}

[1] Takashima Chunsheng. Curved beam bridge. Beijing: China building industry press, 1979.

[2] Fang Cun-Ren. Circular crossing anisotropy For bending of a fanshaped flat plate. The paper sets of Civil Engineering Society, No.82, 1962.

[3] Lingsen Yao, Xinpin Li. A practical method for calculating curved multigirder bridge. China Civil Engineering Journal, 1986,vol.19, No.3, CCES,43p.

[4] Wancun Xie. Space calculation of curved beam bridge with lateral variable stiffness. Shanghai: The proceeding of the Third Annual Conference of the CCES, 1986,306P.

[5] Lingsen Yao, Jiejung Wang. Analysis of curved multi-girder bridge with various sections. China Civil Engineering Journal. 1987, Vol. 20. No.3, CCES, 44p.

[6] Lichu Fan. Bridge Engineering [M]. Beijing: China Communications Press, 2012.

[7] Zhilun Xu. Elasticity(2) [M]. Higher Education Press, 2016. 